\title{
REALIDAD AUMENTADA Y EDUCACIÓN: ANÁLISIS DE EXPERIENCIAS PRÁCTICAS
}

\section{AUGMENTED REALITYAND EDUCATION: ANALYSIS OF PRACTICAL EXPERIENCIES}

\author{
Carlos Prendes Espinosa \\ carlos.prendes@murciaeduca.es \\ Consejería de educación de la Región de Murcia. IES Beniaján. \\ Departamento de informática y comunicaciones. Avenida de Monteazahar s/n 30570, \\ Beniaján, Murcia (España)
}

La realidad aumentada es una prometedora tecnología, ya presente en muchas aulas, que puede ayudar a mejorar el proceso de enseñanza-aprendizaje. El objetivo de este artículo es presentar una recopilación de proyectos llevados a cabo en centros educativos en los últimos años que sirva como perspectiva general del estado del arte de la aplicación de la tecnología de realidad aumentada en el ámbito de la educación en España. Esta recopilación se ha obtenido a través de una investigación documental en revistas especializadas, bases de datos, catálogos on-line y referencias de Internet.

Palabras clave: Realidad Aumentada, educación, informe.

Augmented reality is a promising technology, already present in many classrooms, which can help improve the teaching-learning process. The objective of this paper is to present a compilation of projects carried out in schools in recent years as a general overview of the state of art of the application of augmented reality technology in the field of education in Spain. This compilation was obtained through documentary research on journals, databases, on-line catalogues and Internet references.

Keywords: Augmented Reality, education, report. 


\section{1. ¿Qué es la Realidad Aumentada?.}

La Realidad Aumentada, a partir de ahora RA, es una tecnología que superpone a una imagen real obtenida a través de una pantalla imágenes, modelos 3D u otro tipo de informaciones generados por ordenador. Un ejemplo muy claro e ilustrativo de este tipo de escenarios lo tenemos en conocidos videojuegos como Invizimals (ver https:// www. youtube.com/watch $\mathrm{v}=3 \mathrm{~N} 7 \mathrm{e}$ X5FYKYU\#t=353) o transformers (ver https:/ /www.youtube.com/watch?v=SNfJUIwosFY) que aplican esta tecnología.

Los proyectos etiquetados como realidad aumentada no han parado de crecer en los últimos años. Existen multitud de definiciones de la realidad aumentada y todas aportan algo interesante a la caracterización de este tipo de tecnología. En el informe sobre realidad virtual de Durlach y Mavor (1995) se habla de la RA como sistemas en los cuales entornos reales y virtuales se combinan, aunque esta definición es sencilla adolece de ciertas carencias ya que nos llevaría a catalogar algunos sistemas de RA cuando realmente no lo son. Tal y como ejemplifica Reinoso (2013), si pensamos en la previsión del tiempo que se ofrece en la televisión todos los días; ¿es RA ese mapa sobre el que el presentador señala el tiempo que va a hacer en cada parte del país? La respuesta es que no, aunque podría caber en lo que nos ofrece esta primera aproximación.

Otros autores ofrecen elaboraciones del concepto más complejas que contienen más elementos de discernimiento. Así por ejemplo De Pedro (2011) explica la RA como «aquella tecnología capaz de complementar la percepción e interacción con el mundo real, brindando al usuario un escenario real aumentado con información adicional generada por ordenador. De este modo, la realidad física se combina con elementos virtuales disponiéndose de una realidad mixta en tiempo real» (p. 301). En esta definición, mucho más amplia, observamos la aparición de conceptos de gran trascendencia en los entornos de RA como son la interacción, la realidad mixta o el tiempo real.

Uno de los gurús de la RA da lo que él delimita como una definición funcional de la RA (únicamente se fija en para qué sirve la RA) y define la RA como objetos virtuales o anotaciones que pueden ser superpuestos en el mundo real como si realmente existieran (Kato, 2010). Aunque sencilla y fácil de entender, esta definición de RA incluye los mismos elementos que la anterior pero comprimidos en esa expresión de como si realmente existieran.

Por su parte Basogain, Olabe, Espinosa, Rouèche y Olabe (2007) afirman que «la realidad aumentada no reemplaza el mundo real por uno virtual, sino al contrario, mantiene el mundo real que ve el usuario complementándolo con información virtual superpuesto al real. El usuario nunca pierde el contacto con el mundo real que tiene al alcance de su vista y al mismo tiempo puede interactuar con la información virtual superpuesta» (p.1).

Otros autores se fijan más en qué tipo de hardware o dispositivos se pueden utilizar para los sistemas de RA. Así Fombona, Pascual y Madeira (2012) afirman: «la realidad aumentada amplía las imágenes de la realidad, a partir de su captura por la cámara de un equipo informático o dispositivo móvil avanzado que añade elementos virtuales para la creación de una realidad mixta a la que se le han sumado datos informáticos» (p.203).

Está claro que si se analizan todas estas aportaciones de los diferentes autores se obtiene una imagen bastante clara de lo que 
es la RA, pero sin duda si se quiere una definición técnica, completa y precisa de RA debemos atenernos a la ya clásica que otro de los gurús de la RA dio en su famoso artículo de 1997 «A survey of augmented reality». En este artículo Azuma (1997) nos dice textualmente lo siguiente: «[...] para evitar limitar la realidad aumentada a tecnologías específicas, este artículo define la RA como sistemas que tienen las tres siguientes características:1) Combina lo real y lo virtual. 2) Interactiva y en tiempo real. 3) Registrada en 3D» (p.356).

Estas tres características delimitan de forma clara lo que es o no es un sistema de RA. Específicamente se excluyen los sistemas 2D y se obliga a la interactividad en tiempo real: el usuario debe poder provocar acciones en el entorno y que el entorno se vea modificado y se lo haga saber a su vez al usuario.

\subsection{Niveles de la RA.}

Hay varios autores que hablan de niveles de RA (Estebanell, Ferrés, Cornellà \& Codina, 2012; Lens-Fitzgerald, 2009; Reinoso, 2012; Rice, 2009). Se pueden entender los niveles como una forma de medir la complejidad de las tecnologías involucradas en el desarrollo de sistemas de RA. En principio, a más nivel, mayores son las posibilidades de las aplicaciones. Entre los diferentes autores hay algunos cambios de criterio en cuanto a los niveles que presentan y dónde caería alguna de las tecnologías que se comentan. En este punto es de destacar, por ejemplo, que tanto Reinoso (2012) como Estebanell et al. (2012) introducen la activación mediante imágenes como realidad aumentada markerless mientras que Lens-Fitzgerald (2009) considera este tipo de RA como nivel 1 (no markerless). Rice (2009) incluso advierte de que el término markerless fue utilizado más que nada como una campaña de marketing por parte de las empresas para distinguir el uso de imágenes del uso de las marcas o patrones predefinidos en la activación de las aplicaciones de RA. Para el propósito de este trabajo se ha optado por la utilización del esquema de LensFitzgerald.

Lens-Fitzgerald, el cofundador de Layar, uno de los navegadores de RA más importantes del mundo, escribió un artículo en 2009 donde define los niveles de la RA. En LensFitzgerald (2009) se mencionan cuatro niveles (del 0 al 3). Este autor introduce los códigos $Q R$ (Quick Response) como nivel 0 de RA. Su clasificación queda por tanto de esta manera:

- Nivel 0. Hiperenlazando el mundo físico (physical world hyper linking). Basado en códigos de barra (enlaces 1D, Universal Product Code), códigos 2D (por ejemplo los códigos QR) o reconocimiento de imágenes aleatorias (véase Imagen 1). Lo característico de este nivel 0 es que los códigos son hiperenlaces a otros contenidos, no existe registro en 3D ni seguimiento de los marcadores (básicamente funcionan como un hiperenlace html pero sin necesidad de teclear).

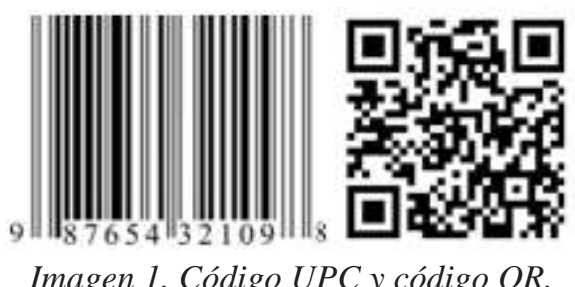

- Nivel 1. AR basado en marcadores (marker based AR). Normalmente es reconocimiento de patrones 2D, el reconocimiento 3D de objetos (por ejemplo, una silla) sería la forma más avanzada de nivel 1 de AR (véase Ima- 
gen 2). Según Estebanell et al. (2012): «los marcadores son unas imágenes en blanco y negro, generalmente cuadradas, con dibujos sencillos y asimétricos» (p.282).

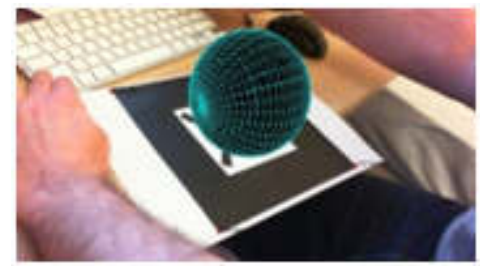

Imagen 2. Ejemplo de modelo 3D asociado a un marcador. Extraída de http:// howtofoldandotherstuff.wordpress.com/ category/game-programming/

- Nivel 2. RA sin marcadores (markerless AR). Mediante el uso del GPS y la brújula de los dispositivos electrónicos conseguimos localizar la situación y la orientación y superponer POI (puntos de interés) en las imágenes del mundo real (véase Imagen 3). LensFitzgerald (2009) lo define como AR basada en GPS-brújula. También puede incluir el uso de acelerómetros para calcular la inclinación.

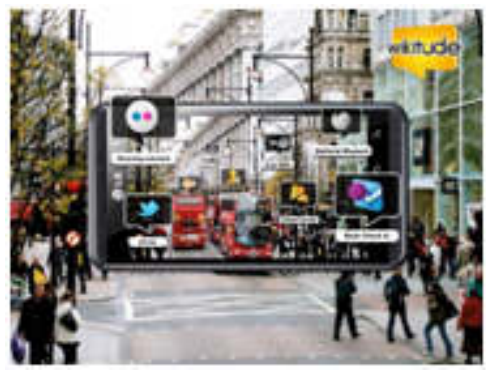

Imagen 3. Ejemplo de RA markerless geoposicionada.Extraída de http:// www.androidcentral.com/lg-and-wikitudeteam-launch-3d-augmented-reality-browser

- Nivel 3. Visión aumentada, citando a Rice (2009):«Debemos despegarnos del monitor o el display para pasar a ligeros, transparentes displays para llevar encima (de una escala como las gafas). Una vez la RA se convierte en VA (visión aumentada), es inmersiva. La experiencia global inmediatamente se convierte en algo más relevante, contextual y personal. Esto es radical y cambia todo» (párr.6). Este nivel no está todavía disponible.

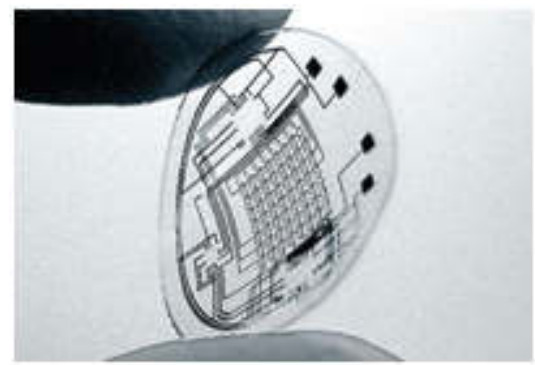

Imagen 4. Lentillas de alta tecnología para su uso como displays. Extraída de http:/ /www.taringa.net/posts/videos/6285450/ Vision-aumentada-Lentes-de-contactoHigh-Tech.html

Rice (2009) incluso habla de un nivel 4 (véase Imagen 4) donde terminaremos usando «displays de lentes de contacto y/o interfaces directos al nervio óptico y el cerebro. En este punto, múltiples realidades colisionarán, se mezclarán y terminaremos con Matrix» (párr.13). Rice habla de un entorno donde podremos olvidarnos de nuestros cuerpos y que será la culminación de la realidad virtual. Por supuesto este apunte en estos momentos es sólo una suposición para un futuro a muy largo plazo aunque ya existen proyectos para producir lentes de contacto que actúen como displays. La tecnología que Reinoso (2013) y otros denominan markerless (entendida como el uso de imágenes como activadores), aunque parecida a la de marcas, es mucho más robusta frente al movi-

Píxel-Bit. Revista de Medios y Educación. No 46. Enero 2015. ISSN: 1133-8482. e-ISSN: 2171-7966. doi: http://dx.doi.org/10.12795/pixelbit.2015.i46.12 
miento, inflexiones y cambios en la iluminación, de la posición y a cambios extremos de perspectiva u oclusión de las imágenes que sirven de marcadores (Ortega, 2013) por lo que su resultado final es de mayor calidad.

\subsection{Relevancia de la tecnología de RA en educación.}

En tanto en cuanto esta tecnología es obviamente prometedora en cuanto a sus previsibles resultados como bien defienden varios autores (De Pedro \& Martínez, 2012; Kato, 2010; Reinoso, 2012), no podemos perder de vista la siempre difícil aplicación de las innovaciones al entorno real escolar. El propio Reinoso (2012), aun reconociendo el enorme potencial de las herramientas que nos proporciona la RA, expresa sus dudas a la hora de implementarlas como herramientas de trabajo en el aula, calificando tal implementación como un desafío. El elemento motivacional, tan importante en la educación parece garantizado, pues como dice Reinoso (2012): «numerosas han sido las investigaciones que sugieren que la RA refuerza el aprendizaje e incrementa la motivación por aprender» ( $\mathrm{p}$. 371).

Es de gran importancia escoger bien los objetivos a conseguir con la utilización de estos sistemas y tener en cuenta a qué audiencia nos dirigimos, como bien afirma Kaufmann (2003): «la Realidad Aumentada no puede ser la solución ideal para todas las necesidades de las aplicaciones educativas pero es una opción a considerar» (p. 1).

Adell y Castañeda (2012) consideran que hay que ser prudentes ante el cambio revolucionario prometido por las tecnologías y que hay que mantener «un cierto grado de escepticismo» (p. 31). En la línea de los mismos autores también sería una cuestión a discutir si estas nuevas innovaciones tecnológicas producen a su vez novedades en la forma de enseñar, lo que denominan pedagogías emergentes. Adell y Castañeda (2012) definen las pedagogías emergentes como: «conjunto de enfoques e ideas pedagógicas [...] que surgen alrededor del uso de las TIC en educación y que intentan aprovechar todo su potencial comunicativo, informacional, colaborativo, interactivo, creativo e innovador en el marco de una nueva cultura del aprendizaje» (pp. 22-23). Cuestión a discutir es si una innovación pedagógica es completamente necesaria hablando de las tecnologías de realidad virtual, Hanson y Shelton (2008) nos ofrecen un enfoque en el cual esto no es necesario: «ofrecemos una reflexión sobre los desafíos del diseño y desarrollo de estas aplicaciones a un nivel personal, y ofrecemos recomendaciones sobre un enfoque que puede tener sentido para usar RV en una práctica instruccional tradicional» (p.118). Según estos autores por tanto estas tecnologías pueden ofrecer ventajas incluso integrándose en métodos de enseñanza tradicional.

Otros autores muestran un sesgo más optimista y se muestran más convencidos del uso de la RA en el aula, así Estebanell et al. (2012) habla que las tecnologías en dispositivos móviles superan la limitación del tiempo y del espacio en los entornos de aprendizaje pero además añaden que las aplicaciones de RA «no solo responden a este tipo de exigencia si no que la amplían de manera cualitativamente significativa al ofrecer información situada, contextualizada, desde el lugar y en el momento que el consumidor la precisa» (p. 290). Por su parte Billinghurst (2002), creador del primer libro de RA «Magic book»nos da muy buenas razones pensar que la RA podría ser muy valiosa en la educación: 
«Soporte de una fluida interacción entre el entorno real y el virtual.El uso de la metáfora de la interfaz tangible para la manipulación de objetos. La habilidad para hacer suavemente la transición entre realidad y virtualidad» (p. 2).

En la misma línea González (2013) da otras tres razones para apostar por la RA: «Posibilita contenidos didácticos que son inviables de otro modo. Nos ayuda a que exista una continuidad en el hogar.Aporta interactividad, juego, experimentación, colaboración, etc.» (p. 1).

Roussou (2004) nos proporciona un enfoque metodológico que considera adecuado aplicar a los entornos de realidad virtual, tales argumentos pueden ser igualmente compatibles con los sistemas de RA. De hecho la autora reconoce la dificultad, debido a su elevado coste, de implementaciones de RV que sirvan a los objetivos educativos y que por tanto estarían en principio restringidos a museos o instituciones de investigación, pero que esta limitación se verá superada (como de hecho ya lo está siendo) por los avances que desde la fecha del artículo se han producido en los entornos tecnológicos y redes de acceso.

La autora dice que «el constructivismo se adopta como base para el desarrollo de entornos altamente interactivos y participativos, donde el usuario es capaz de modificar, construir, probar ideas, e involucrarse activamente en la resolución de un problema» (p. 5). Roussou (2004)afirma también que hay un gran consenso entre los educadores sobre que la interactividad en la enseñanza es necesaria y sigue el argumento de G.R. Amthor de que «la gente retiene [...] el 75\% de lo que ve, oye y hace» (pp. 3-4). Desde este punto de vista parece claro que la tecnología de RA ayudará al proceso de aprendizaje de los alumnos debido entre otras razones al alto grado de interacción que proporciona. Esta forma de enseñanza es el conocido como enfoque «aprender haciendo» (learning through activity o learning by doing) también conocido como aprendizaje activo (basado en actividades, proyectos, etc.) y «aprender jugando» (learning through play), lo cual es compatible con el punto de vista constructivista que defiende la autora.

\section{Metodología.}

A la hora de afrontar el estudio de este tema la falta de referencias encontradas ha sido una gran dificultad. Hay una gran cantidad de ellas sobre RA pero el número disminuye drásticamente cuando hablamos de forma específica de RA aplicada a la educación. En estas referencias los análisis y disertaciones se refieren a diferentes tipos de tecnologías de RA aplicables a la educación, experiencias basadas en RA en entornos educativos y opiniones que los autores describen sobre el futuro de estas tecnologías y su aplicabilidad pero la bibliografía adolece de falta de estudios de investigación sobre la idoneidad de estas tecnologías, metodología, estudios cuantitativos y conclusiones basadas en fuentes primarias sobre el objeto de estudio.

Esta falta de información, producida sin duda por el hecho de la novedad y reciente aparición en nuestra vida diaria de la RA, hizo que lleváramos a cabo una investigación documental sobre RA y educación con carácter exploratorio para poder abordar propuestas de buenas prácticas apoyadas en estas tecnologías.

La investigación exploratoria en la que se basa este artículo sigue los postulados de (Arias,2006; Hernández, Fernández \& Baptista, 2003; Selltiz,Wrightsman, Cook \& 
Balch, 1980). Por su parte Arias (2006) define la investigación exploratoria como la que «se efectúa sobre un tema u objeto desconocido o poco estudiado, por lo que sus resultados constituyen una visión aproximada de dicho objeto, es decir, un nivel superficial de conocimientos» (p. 23).

Este estudio exploratorio, siguiendo a Selltiz et al. (1980), podrá ser utilizado para dirigir la formulación más precisa de problemas de investigación después de que las conclusiones ofrecidas por él permitan tener un conocimiento más preciso acerca del tema tratado. Esta misma idea es corroborada por Hernández et al. (2003) cuando afirman: «los estudios exploratorios nos sirven para aumentar el grado de familiaridad con fenómenos relativamente desconocidos, obtener información sobre la posibilidad de llevar a cabo una investigación más completa» (p. 70). En el caso que nos ocupa se lleva a cabo un proceso de búsqueda documental y obtención de datos descriptivos sobre RA mediante la revisión de la información encontrada en la literatura.

Por tanto este artículo expone los resultados de una investigación documental definida en la línea de Arias (2006) como «proceso basado en la búsqueda, recuperación, análisis, crítica e interpretación de datos secundarios, es decir, los obtenidos y registrados por otros investigadores en fuentes documentales: impresas, audiovisuales o electrónicas» (p. 27).

\subsection{Objetivo de la investigación.}

El objetivo de la investigación es por una parte averiguar la tipología de las actividades que se realizan en centros educativos, fundamentalmente españoles, pero también conocer la tecnología de RA que utilizan, el software y la metodología, todo ello en orden aanalizar las experiencias relevantes en este campo y conocer el estado del arte para poder definir nuevas líneas de investigación y/ o actividades relacionadas con la RA adaptadas al contexto educativo propio.

\subsection{Procedimiento.}

El proceso de búsqueda de referencias relacionadas con el tema comienza con las revistas especializadas, para ello se consulta el índice de impacto de las revistas de ciencias sociales (IN-RECS) elaborado por el grupo de investigación EC3 (Evaluación de la Ciencia y de la Comunicación Científica) de la Universidad de Granada (ver http:// ec3.ugr.es/layout.php?id=miembros). El índice de impacto está disponible públicamente en la web http://ec3.ugr.es/ec3/ Educacion.html y el último año accesible es el 2011 que es el utilizado para obtener las revistas especializadas en educación y/o tecnología educativa más relevantes.

En la búsqueda documental se han utilizado, siempre que ha sido posible, los propios servicios de búsqueda web de las revistas con los términos «Realidad Aumentada», «Augmented Reality» $y$ «Realitat augmentada», ya que se han detectado algunas revistas editadas en catalán. En aquellas revistas en las que no ha sido posible una búsqueda indexada, se ha realizado una revisión que podríamos calificar de «manual» accediendo a los sumarios de todos los números y comprobando las referencias existentes al tema de la tecnología de RA. Mientras que las búsquedas electrónicas accedían a todo el catálogo de las publicaciones, en la revisión manual se han considerado los números de las revistas cuya fecha de publicación fuese el 2008 o posterior. Esta fecha es 
anterior a la aparición en el mercado del primer navegador de RA. Este criterio se ha seleccionado debido a que las aplicaciones de RA geolocalizadas o en entornos abiertos necesitan de este tipo de herramientas para funcionar, con lo que la búsqueda a partir de esa fecha debe cubrir todas las experiencias de ese tipo. Por otro lado es muy posible que existan actividades con códigos QR antes de esa fecha pero la escasez de información sobre este tipo de actividades permite suponer que la acotación de la búsqueda utilizando ese límite temporal no ha acarreado una pérdida significativa de información relevante.

Posteriormente a la búsqueda en revistas especializadas, se han realizado búsquedas en catálogos on-line sobre artículos de Realidad Aumentada con los mismos términos utilizados para las revistas. Se realiza una búsqueda en las bases de datos bibliográficas del CSIC (Consejo Superior de Investigaciones Científicas), concretamente se utilizan los sumarios ICYT de Ciencia y tecnología, IME de biomedicina e ISOC de ciencias sociales y humanidades. Se revisa también el catálogo on-line de Dialnet (Universidad de la Rioja).

La siguiente fase de búsqueda se ha llevado a cabo en Internet, concretamente en el buscador de Google utilizando los mismos términos de búsqueda ya explicitados. La cantidad de referencias ha sido bastante grande y se ha tenido realizar una labor de filtrado muy trabajosa aunque con estas búsquedas se ha podido acceder a algunos recursos (como por ejemplo conferencias de gurús sobre RA en diferentes congresos grabadas en Youtube) que hubieran sido imposibles de localizar mediante otros métodos de búsqueda. En esta etapa ha tenido también especial importancia la revisión de documentación disponible en red de los diferentes congresos sobre RA.
Se debe indicar que la búsqueda documental a la que se refiere este apartado se circunscribe a recursos españoles puesto que el objetivo del artículo, como se indica en el resumen, es la revisión de experiencias en el ámbito educativo español. Esto no es óbice para que, como se puede comprobar en el listado de las fuentes bibliográficas, el marco teórico haya sido desarrollado tras una exhaustiva búsqueda de recursos en inglés y español de autores e instituciones internacionales.

\subsection{Población y muestra.}

En la revisión de las revistas especializadas se utilizan los datos de los dos primeros cuartiles, obteniéndose un total de 58 publicaciones revisadas según los criterios expuestos. En estas revistas se registran un total de 13 referencias para RA de las cuales sólo 4 artículos son de utilidad para la investigación, el resto mencionan la RA de pasada o no incluyen ninguna experiencia.

En la revisión de las bases de datos del CSCI el resultado total es de 48 documentos relacionados con los términos de realidad aumentada. Realizando sobre estos documentos la pertinente revisión detallada se observan muchas referencias sobre entornos de RA en museos y yacimientos arqueológicos y entornos abiertos pero menos en referencia a nuestra área de interés particular (aplicaciones de RA para uso educativo). La búsqueda en el catálogo on-line Dialnet obtiene para la búsqueda «realidad aumentada» 648 referencias, al refinar la búsqueda se introduce el término «educación» obteniendo 84 referencias revisadas algunas de las cuales han sido incluidas en este artículo. 
FICHA DESCRIPTIVA DE ACTIVIDAD UTILIZANDO REALIDAD AUMENTADA

\begin{tabular}{|c|c|c|}
\hline \multirow{7}{*}{$\begin{array}{l}\text { Datos básicos de } \\
\text { referencia }\end{array}$} & \multicolumn{2}{|l|}{ Centro educativo $^{(1)}$ : } \\
\hline & \multicolumn{2}{|l|}{ Página $w_{e b}^{(2)}$ : } \\
\hline & \multicolumn{2}{|l|}{ Ciudad $^{(3)}$ : } \\
\hline & \multicolumn{2}{|l|}{ Etapa educativa $^{(4)}$ : } \\
\hline & \multicolumn{2}{|l|}{$\operatorname{Ciclos}^{(5)}$ : } \\
\hline & \multicolumn{2}{|l|}{ Curso y grupos ${ }^{(6)}$ : } \\
\hline & \multicolumn{2}{|l|}{ Curso académico ${ }^{(7)}$ : } \\
\hline \multirow{12}{*}{$\begin{array}{l}\text { Datos sobre la } \\
\text { actividad }\end{array}$} & \multicolumn{2}{|c|}{ Fuentes de información sobre la actividad ${ }^{(8)}$ : } \\
\hline & \multicolumn{2}{|c|}{ Número total de alumnos implicados en la realización de la actividad ${ }^{(9)}$ : } \\
\hline & \multicolumn{2}{|c|}{ Utilización de nuevas tecnologías complementarias ${ }^{(10)}$} \\
\hline & \multicolumn{2}{|l|}{ Datos de los profesores ${ }^{(11)}$ : } \\
\hline & \multirow{6}{*}{\multicolumn{2}{|c|}{$\begin{array}{l}\text { Descripción de la actividad }{ }^{(12)} \text { : } \\
\text { Objetivos } \\
\text { Contenidos } \\
\text { Agrupamiento (individual, parejas, en grupo, otros) } \\
\text { Metodología } \\
\text { Evaluación }\end{array}$}} \\
\hline & & \\
\hline & & \\
\hline & & \\
\hline & & \\
\hline & & \\
\hline & \multicolumn{2}{|c|}{ Fechas de realización de la actividad $^{(13)}$ : } \\
\hline & \multicolumn{2}{|c|}{ Lugares de realización de la actividad $^{(14)}$ : } \\
\hline \multirow{5}{*}{$\begin{array}{c}\text { Análisis de la } \\
\text { tecnología de RA } \\
\text { implicada }\end{array}$} & \multicolumn{2}{|l|}{ Nivel de $\mathrm{RA}^{(15)}$ : } \\
\hline & \multicolumn{2}{|c|}{ Clasificación de la actividad en un subtipo de RA ${ }^{(16)}$ : } \\
\hline & \multirow{3}{*}{$\begin{array}{l}\text { Descripción de los medios } \\
\text { utilizados }\end{array}$} & Software ${ }^{(17)}$ : \\
\hline & & Hardware $^{(18)}$ : \\
\hline & & $\begin{array}{l}\text { Conexión a Internet }{ }^{(19)}(\text { sí,no): } \\
\text { Redes Wifi. } \\
\text { Redes de telefonía móvil. } \\
\text { Otras. }\end{array}$ \\
\hline \multirow[t]{2}{*}{ Evaluación } & \multicolumn{2}{|c|}{ Datos disponibles sobre evaluaciones del personal participante ${ }^{(20)}$ : } \\
\hline & \multicolumn{2}{|c|}{ Datos disponibles sobre evaluaciones externas y difusión de la actividad ${ }^{(21)}$ : } \\
\hline
\end{tabular}

Tabla 1. Ficha de análisis.

$-195-$

Píxel-Bit. Revista de Medios y Educación. No 46. Enero 2015. ISSN: 1133-8482. e-ISSN: 2171-7966. doi: http://dx.doi.org/10.12795/pixelbit.2015.i46.12 


\subsection{Instrumento de análisis de experien- cias con RA.}

Para el análisis de las experiencias se utilizó una ficha validada por juicio de expertos (del Grupo de Investigación en Tecnología Educativa de la Universidad de Murcia). Se incluye a continuación en la Tabla 1 la ficha en su versión final.

\section{Resultados.}

En este punto se plasma la información obtenida de la búsqueda documental. Las experiencias que se presentan en este punto se seleccionan atendiendo a dos criterios fundamentales: la información disponible acerca de la experiencia y la tipología de las actividades de RA llevadas a cabo. El primer criterio es relevante por cuanto la mayoría de las experiencias analizadas ofrecen poca o muy poca información para completar la ficha de análisis y las fuentes documentales de las presentadas en este resumen sí permiten la elaboración, al menos parcial, de tal análisis, como se recoge en Prendes (2014). Por otro lado, aunque algunas de las actividades presentadas adolezcan también de falta de información, son de importancia en la medida en que completan la tipología de actividades de RA, ofreciendo una visión completa acerca de las posibilidades de esta tecnología en educación.

La información presentada aquí ofrece ejemplos de cada uno de los diferentes tipos de proyectos que son factibles hoy en día para su aplicación real. Los proyectos que se comentan en esta sección cumplen con los requisitos de ser aplicables por cuestiones de equipamiento tecnológico y disponibilidad de software y hardware adecuado.
El primer tipo de proyectos de RA son los libros didácticos de Realidad Aumentada; en este apartado se pueden mencionar los libros de Aumentaty, disponibles en su página Web http://www.ar-books.comdonde podemos ver un libro de tecnología de cuarto de la ESO hecho con Realidad Aumentada junto a otras obras.

En la misma línea de proyectos educativos la empresa Aumentaty proporciona el software realitat3, que contiene multitud de objetos de Realidad Aumentada de utilidad para la docencia. Citando a Vian (2011): «para LabHuman, el sistema de RA ofrece a los alumnos por una parte, mejor entendimiento y visión de las asignaturas educativas más complejas y por otra parte, les dota de mayor motivación para el estudio. En cuanto al profesorado, el sistema se adapta a las necesidades pedagógicas que tengan» (p.103). Es de destacar además que este software ya ha sido probado con éxito en centros educativos reales (colegio San Cristóbal de Castellón) y tanto profesores como alumnos han mostrado su satisfacción por los resultados obtenidos (Vian, 2011).

Diego (2014) lleva a cabo una experiencia con alumnos de tercer ciclo de educación primaria para generar un libro de RA (el autor lo denomina guía visual) y una aplicación geolocalizada sobre la península de la Magdalena (Santander) utilizando Layar creator como software de RA además de software auxiliar para edición de imágenes, textos, grabación de sonidos, etc.

Sánchez (2011) realiza una experiencia en el instituto SEK de Ciudalcampo donde utiliza un entorno con BuildAR y el generador de modelos 3D 3DSMax para conseguir que los alumnos generen sus propios cuadernillos de modelos de RA en el sistema de dibujo axonométrico. Esta profesora además utiliza 
la utilidad Dropbox para promocionar el trabajo colaborativo. Los proyectos relacionados con el dibujo técnico son muy adecuados para la RA ya que suponen un manejo de las capacidades espaciales en el que la RA ha demostrado su utilidad educativa.

Es de señalar también la importancia de los videojuegos como uno de los campos más productivo de la Realidad Aumentada (National Academy of Sciences, 2012). En este sentido cabe destacar los proyectos que mezclan juegos y educación, el área conocida como edutainment (Portalès, 2008). Como ejemplos de estas aplicaciones Reinoso (2012) menciona tres: Environmental detectives del Instituto Tecnológico de Massachusetts (MIT), proyecto enredaMadrid (Telefónica) y Libregeosocial (Universidad Rey Juan Carlos).

El proyecto EspiRA, tal y como menciona Reinoso (2012) «es un proyecto de Geolocalización y Realidad Aumentada [...] que pretende proporcionar una plataforma que sea utilizada de manera sencilla e intuitiva en el mundo educativo, permitiendo que profesores y alumnos puedan acceder fácilmente a esta tecnología» (p. 390). Cabe destacar dentro de este tipo de proyectos los que menciona Silva (2013) sobre códigos QR asociados a las calles de la localidad de Rubí (Barcelona) y utilizando POI y geoposicionamiento para asociar información virtual (en un blog) a los puntos del municipio donde se produjo una riada en los años 50. Tal como recoge Silva (2013) el proyecto de códigos QR fue galardonado con el Primer Premio Internacional Educared 2011 al uso de la tecnología celular en el ámbito educativo.

Otro software útil para aplicar en proyectos educativos es Eduloc (ver http:// www.eduloc.net/). Este tipo de aplicaciones caerían dentro del rango de geolocalización.
Reinoso (2012) identifica seis aplicaciones significativas de RA en educación y para cada una de ellas nos proporciona un ejemplo:

a) Aprendizaje basado en el descubrimiento. Reinoso nos propone la aplicación historypin que es otra aplicación basada en geolocalización para mostrar imágenes históricas de los lugares (POI, Points OfInterest) donde los usuarios de la aplicación han querido mostrar una imagen.

b) Desarrollo de habilidades profesionales. «La formación profesional es una de las grandes áreas de aplicación de la R.A., permitiendo mejorar la comprensión en actividades de formación práctica y recrear situaciones reales de trabajo» (p. 375). Reinoso nos presenta el proyecto Visir de la universidad de Deusto. Un cuaderno de prácticas de laboratorio aumentado con la información de los instrumentos necesarios para la realización de la práctica.

c) Juegos educativos con RA.

d) Modelado de objetos 3D. Reinoso (2012) lo define como: «mediante herramientas de modelado de objetos y aplicaciones de R. A., el alumno puede crear y visualizar modelos 3D y manipularlos: acercarlos, alejarlos, girarlos, colocarlos en lugares determinados o explorar sus propiedades físicas» (p. 380). Aquí cabe mencionar software como AR-media plugin for sketchup, BuildAR Free Version o aplicaciones como A-RA de Víctor Valbuena que comentaremos posteriormente.

e) Libros con RA. Ya mencionados también anteriormente.

f) Materiales didácticos. Entre los que nos propone Reinoso están la colección de modelos (ver http://www.catedu.es/ webcatedu/index.php/descargas/realidadaumentada) de RA del Centro Aragonés para las Tecnologías de la Educación (CATEDU) 
o el proyecto realitat3 de LabHuman ya mencionado también anteriormente. Según Reinoso (2012): «la oferta de este tipo de recursos es aún escasa, sería deseable el desarrollo de plataformas y aplicaciones educativas que permitan al profesorado crear y compartir materiales propios con R.A. de una forma sencilla» (p. 389).

Arbúes (2013) nos presenta proyectos reales llevados a cabo con herramientas de realidad aumentada en el IES Vilaratza (Vilassar de Mar, Barcelona) por los propios alumnos del instituto. Entre ellos cabe destacar el de Víctor Valbuena A-RA, disponible en http:// www.sacosta.org/vv/, y audioguías creadas con la herramienta woices (otra aplicación de geolocalización para archivos de audio).

Arbúes (2013) también nos comenta el uso de códigos $Q R$, incluso códigos $Q R$ con voz. Dentro de esta categoría de aplicaciones (uso de códigos $Q R$ ) podemos mencionar experiencias como las de la profesora Eva María Perdiguero en el uso de códigos QR en clase de matemáticas (ver http:// evamate.blogspot.c om.es/2013/06/codigosqr-y-realidad-aumentada-en-1-de.html) así como los usos que describe Gamboa (2012) en el IES La Rosaleda de Málaga o los descritos en el blog http://qredu.net/blog/. Un ejemplo muy curioso de aplicación de RA usando códigos QR es el que presenta Pérez-Fuentes, Álvarez-Bermejo, Molero, Gázquez \& López (2011), en esta aplicación, llamada VERA(Violencia Escolar y Rendimiento Académico) se utiliza la RA para comprobar el patrón de interacción entre los alumnos para estudiar y corregir comportamientos de violencia escolar en las aulas. Como último ejemplo de la gran cantidad de experiencias con códigos QR disponibles se mencionará a Izquierdo (2013) que presenta una aplicación QR para el aprendizaje de rutinas de calenta- miento en español y alemán en el marco de un programa de intercambio de alumnos de primero a tercero de la ESO.

De Pedro y Martínez (2012) presentan ejemplos del uso de RA en educación primaria para el área de las matemáticas y en la educación superior en los ámbitos de:

a) Arquitectura. En la Escuela de Arquitectura y Urbanismo de la Universidad de Chile

b) Dibujo técnico. Mediante el uso de Google SketchUp y el plugin AR-media.

c) Laboratorios de ingeniería. En la Escuela Superior de Ingeniería de Bilbao (España) donde según De Pedro y Martínez (2012): «los estudiantes pueden examinar el funcionamiento de dispositivos eléctricos o mecánicos reales, como máquinas eléctricas, circuitos electrónicos, modelos a escala, actuadores neumáticos, motores, etc.» (p. 104).

d) Matemáticas. La Universidad EAFIT (Colombia) tiene un proyecto de RA para la comprensión del cálculo de varias variables.

Como última categoría de ejemplos de aplicaciones de RA mencionamos las utilizadas en museos de las que existen gran variedad de ejemplos, como muestra se menciona a Perales y Adam (2013) que describen dos de tales aplicaciones: una basada en códigos QR y otra aplicable a imágenes de gran definición donde se ofrecen anotaciones sonoras en determinados puntos de la imagen, por ejemplo, para ofrecer explicaciones detalladas sobre una obra de arte.

\section{Discusión.}

Aun siendo la tecnología lo suficientemente madura para ser útil en los entornos educativos, los nuevos desarrollos de RA llevarán a una nueva generación de aplicaciones 
que desplegarán mucho más el potencial de la Realidad Aumentada. El reconocimiento de objetos 3D (Ortega, 2013) para el reconocimiento de objetos sólidos desde cualquier ángulo y perspectiva, la búsqueda visual y el uso de interfaces HMD wearables del tipo de Google glass aumentarán el valor de los sistemas de RA. Otras mejoras vendrán de la mano de aplicaciones que permitan la interacción entre los propios marcadores, esta clase de interacción ya está conseguida y funciona perfectamente (por ejemplo, ARChemistry de Paradox). No se ha encontrado ninguna aplicación gratuita que las implemente, ésta es una de las características que la empresa Aumentaty ha señalado para desarrollar en su próxima versión de su software gratuito Aumentaty Author (Anguita, 2013).

La Realidad Aumentada es una de las tecnologías que está llamada a quedarse instalada en nuestras vidas. Uno de los objetivos de los miembros del sitio Web http:// augmentedworldexpo.com/augmentedrealityorges conseguir mil millones de usuarios de RA en 2020. Prácticamente todos los días surgen aplicaciones nuevas y nuevas ideas para ser implementadas y esta fuerza innovadora crecerá con los dispositivos wearables. Para darse una idea, una búsqueda en el repositorio de aplicaciones del sistema Android de Google (playStore) del término augmented reality nos devuelve más de doscientas aplicaciones diferentes y subiendo casi cada día.

Los elementos motivacionales del uso de la tecnología están ya suficientemente probados como para quedar fuera de discusión (Reinoso, 2012) y muchos autores defienden que la tecnología de RA sirve realmente para mejorar la práctica educativa y la comprensión de ciertos aspectos de la realidad por parte de los alumnos. Igualmente se ha utilizado también para proporcionar prácticas a los discentes (aplicaciones médicas) que de otra forma serían imposibles de proporcionar con sujetos reales.

El mundo del marketing y la publicidad no es ajeno a esta tecnología y como ejemplo podemos ver como la multinacional de muebles IKEA ha lanzado su catálogo con RA para el 2014.

Después de todo lo expuesto y estudiado casi podríamos afirmar con seguridad que seguiremos oyendo hablar mucho de Realidad Aumentada en los próximos años y desde luego en educación no pasará desapercibida. Tal afirmación se ve reforzada por las conclusiones a las que Redondo, Fonseca, Sánchez \& Navarro (2014) llegan en su estudio cuantitativo sobre las ventajas obtenidas con la aplicación de la RA: «se reflejaron tanto en el grado de motivación mostrado por los alumnos [...] como en la mejora en su rendimiento académico. Así, los resultados obtenidos muestran que los grupos que utilizan la nueva metodología (RA-ML) adquieren una mejora en las calificaciones» (p. 167).

En cuanto a las conclusiones que se pueden extraer del propio proceso de investigación se deben señalar algunas limitaciones y fortalezas. Es complicado sacar conclusiones empíricas sobre la idoneidad de las propuestas presentadas a falta de más estudios que aborden la cuestión, lo cual es un campo de estudio abierto para futuras investigaciones. Se debe destacar la amplia revisión de tecnologías y experiencias llevada a cabo y presentada en este artículo.

La información contenida en este artículo sirve como punto de partida para el planteamiento de hipótesis de trabajo, en la línea de Redondo et al. (2014), que se verifiquen mediante estudios cualitativos y/o cuantitativos 
que respondan a preguntas del tipo de cuál es el nivel de RA que consigue mejores resultados según los contenidos a tratar, qué tipos de aplicaciones son más útiles dependiendo de variables cómo el tipo de RA, nivel de los alumnos o nivel de uso de las TIC y, en definitiva, sobre los beneficios del uso de la tecnología de RA en el aula.

\section{Notas.}

Cualquier dato de los abajo descritos si no se dispone de él se deberá dejar indicado en la ficha como NO DISPONIBLE.

(1): Denominación del centro educativo responsable de la realización de la actividad.

(2): Página web del centro educativo y en su caso de información sobre la propia actividad.

(3): Ciudad del centro educativo.

(4): Infantil, primaria, ESO, bachillerato, formación profesional o universidad para el sistema educativo español. Equivalentes para otros sistemas educativos.

(5): Si la etapa educativa está dividida en ciclos indicar aquí el (o los ) ciclos donde se lleva a cabo la actividad (ejemplo segundo ciclo de primaria o primer ciclo de secundaria).

(6): Curso (o cursos) que realizan la actividad y de qué grupos de cada curso son los alumnos que realizan la actividad (por ejemplo, $4^{\circ} \mathrm{A}, 3^{\circ} \mathrm{B}$ y $\left.3^{\circ} \mathrm{C}\right)$.

(7): Curso académico en el que se realiza la actividad (por ejemplo 2011/2012).

(8): Referencias de donde se ha extraído la información de la actividad. Si se ha obtenido a partir de una recogida de datos propia, búsqueda de información o, por ejemplo, mediante entrevistas con los implicados indicarlo también en este apartado.
(9): Número total de alumnos que realizan la actividad (todos los alumnos de todos los grupos).

(10): ¿Se complementa la actividad con el uso de alguna herramienta más? Blog, wiki, redes sociales, página web, etc.La actividad se realiza de forma individual o existe algún tipo de agrupamiento.

(11): Información disponible sobre el profesorado responsable del diseño y realización de la actividad. Principalmente ,si están disponibles, nombre y apellidos, correo electrónico y teléfono de contacto u otros datos.

(12): Descripción lo más detallada posible sobre el diseño y el desarrollo de la actividad. Debe incluir: objetivos, contenidos, agrupamiento, metodología y evaluación. En el agrupamiento se distinguirá entre actividades individuales, en pareja, en grupo (de 3 a 5 alumnos ) u otros tipos.

(13): Fechas en las que efectivamente se realizó la actividad.

(14):Lugares en los que se llevó a cabo la actividad (sobre todo si son diferentes del centro educativo).

(15): Nivel 0, nivel 1 o nivel 2 (el nivel 3 todavía no está disponible).

(16): Cerrada o abierta, local o ubicua, móvil o espacial, individual o colaborativa, de escritorio, portátil o equipamiento especializado y de reconocimiento de formas, geoposicionamiento o híbrida.

(17): Identificación de los sistemas software utilizados incluyendo la plataforma de RA, el sistema operativo y el software de apoyo en su caso.

(18): Hardware utilizado en las actividades, si son varios y no son relevantes para la actividad se indicará aquí (por ejemplo, si se ha utilizado una plataforma basada en ARToolkit con Android utilizando los móviles de los alumnos se indicará como hardware hetero- 
géneo ya que no nos importan exactamente los modelos de móviles Android utilizados).

(19): Conexión a Internet. Referente a los requisitos de conectividad a redes de datos para la actividad utilizados. Si la respuesta fuera que sí (es necesaria la conexión a Internet), se tiene la opción de elegir si el acceso se realiza a través de wifi, redes de datos móviles u otras.

(20): Cualquier información disponible sobre las evaluaciones de la actividad llevadas a cabo por parte del personal participante (profesores, alumnos).

(21): Información sobre cualquier evaluación externa que pueda existir sobre la actividad y qué medios se han utilizado, si alguno, para difundirla.

\section{Agradecimientos.}

A la Dra. Linda Castañedo Quintero y la Dra. Adolfina Pérez i Garcías por la dirección de este trabajo. A la Dra. María Paz Prendes Espinosa por la revisión técnica del contenido de este artículo.

\section{Referencias bibliográficas.}

Adell, J. \& Castañeda, L. (2012). Tecnologías emergentes, ¿pedagogías emergentes?. En J. Hernández, M. Pennesi, D. Sobrino \& A. Vázquez (Coords). Tendencias emergentes en educación con TIC. (pp.18-63). Barcelona: Editorial espiral.

Anguita, E. (2013). GeoAumentaty y Aumentaty Visual Search. [Archivo de vídeo]. Recuperado de https:// www.youtube.com/watch? $\mathrm{v}=\mathrm{A} 9 \mathrm{gYdIdU6}$ 24\&list=PLdi_AmlTYDwoNfCoDClhcT8H QHNyCC0KM\&index $=15$

Arbúes, J. (2013). Realidad Aumentada desde la secundaria. Aplicaciones didácticas. [Archivo de vídeo]. Recuperado d e h t t p : / / w w w. y o u t u b e . c o m / watch? $\mathrm{v}=\mathrm{kySKV} \mathrm{pL}-\mathrm{vh}$ M\&list=PLdi AmlTYDwoNfCoDClhc T8HQHNyCCOKM

Arias, F.G. (2006). El Proyecto de Investigación. Introducción a la Metodología Científica ( $5^{\mathrm{a}}$ edición). Caracas-Venezuela: Editorial Episteme.

Azuma, R. (1997). A Survey of Augmented Reality.Presence: Teleoperators and Virtual Environments, 6 (4), 355-385.

Basogain, X., Olabe, M., Espinosa, K., Rouèche, C.\& Olabe, J.C. (2007, mayo). Realidad Aumentada en la Educación: Una tecnología emergente. Comunicación presentada a Online Educa Madrid 2007: $7^{\text {a }}$ Conferencia Internacional de la Educación y la Formación basada en las Tecnologías,Madrid.

Billinghurst, M. (2002).Augmented Reality in education. New horizons for learning. Recuperado de http://www.it.civil.aau.dk/it/ education/reports/ar_edu.pdf

De Pedro, J. (2011). Realidad Aumentada: un nuevo paradigma en la educación superior. En E. Campo, M. García, E. Meziat \& L. Bengochea (eds.). Educación y sociedad. (pp. 300-307). Chile: Universidad La Serena.

De Pedro, J. \& Martínez, C.L. (2012). Realidad Aumentada: Una Alternativa Metodológica en la Educación Primaria Nicaragüense. IEEE-RITA. 7 (2), 102-108.

Diego, R. (2014). Realidad aumentada en documentos e imágenes. Revista Aula de innovación educativa, 230, 65-66.

Durlach, N.I. \& Mavor, A. S. (Eds.) (1995).Virtual Reality: scientific and technological challenges. Washington, D.C: National Academy Press.

Estebanell, M., Ferrés, J., Cornellà, P. \& Codina, D. (2012). Realidad aumentada y códigos QR en educación. En J. Hernández, M. Pennesi, D. Sobrino \& A. Vázquez (Coords). 
Tendencias emergentes en educación con TIC. (pp. 277-320). Barcelona: Editorial espiral.

Fombona, J., Pascual, M.A. \& Madeira, M.F. (2012). Realidad Aumentada, una evolución de las aplicaciones de los dispositivos móviles. Píxel-Bit. Revista de Medios y Educación, 41, 197-210.

Gamboa, J.L. (2012). El uso de códigos QR en la enseñanza. En J. Hernández, M. Pennesi, D. Sobrino \& A. Vázquez (Coords). Tendencias emergentes en educación con TIC. (pp. 401-425). Barcelona:Editorial espiral.

González, O. (2013). Educación aumentada. Centro de conocimiento de tecnologías aplicadas a la educación (CITA), 19. Recuperado de file://C:/Users/Aguilar/Downloads/ articulos19.pdf

Hanson, K. \& Shelton, B. E. (2008). Design and Development of Virtual Reality: Analysis of Challenges Faced by Educators. Educational Technology \& Society, 11 (1), 118-131.

Hernández, R., Fernández, C. \& Baptista, P. (2003). Metodología de la investigación ( $3^{\mathrm{a}}$ ed.). México: McGraw -Hill.

Izquierdo, A. (2013). Códigos QR flexibles: un proyecto con dispositivos móviles para el trabajo de calentamiento en educación física. EmásF: revista digital de educación física, 4 (23), 53-71.

Kato, H. (2010). Return to the origin of Augmented Reality [Archivo de vídeo]. Presentation at IEEE International

Symposium on Mixed and Augmented Reality 2010 (Seoul, Korea). Panel discussion: «The Future of ISMAR: Converging Science, Business, and Art» (organized by Henry Fuchs and Christian Sandor). Recuperado de h t t p : / / w w w. youtube.com/wat ch ?v=b33eqcVz7X8
Kaufmann, H. (2003). Collaborative augmented reality in education. Proc. Imagina 2003 Conf. (Imagina03), Mónaco. Recuperado de https://www.ims.tuwien.ac.at/ publications/tuw-137414.pdf

Lens-Fitzgerald, M. (2009).Augmented Reality Hype Cycle.Recuperado de http:// www.sprxmobile.com/the-augmented-realityhype-cycle.

National Academy of Sciences (2012). Frontiers of engineering: reports on leading-edge engineering from the 2012 symposium. Washington, DC: National Academy of Engineering.

Ortega, M. (2013). Pasado, presente y futuro de la realidad Aumentada. [Archivo de vídeo]. Recuperado de http:// www.youtube.com/watch?v=Jo81 cmDN nv8\&list=PLdi_AmlTYDwoNf CoDClhcT8H QHNyCCOKM

Perales, V. \& Adam, F. (2013). Integración de GIS (sistemas de georreferenciación de la información) y localización espacial en prácticas pedagógicas y lúdicas vinculadas a museos. Arte, individuo y sociedad, 25 (1), 121-133. doi: http:/dx.doi.org/10.5209/ rev_ARIS.2013.v25.n1.41168

Pérez-Fuentes, M.C., Álvarez-Bermejo, J.A., Molero, M.M., Gázquez, J.J. \& López, M.A. (2011). Violencia Escolar y Rendimiento Académico (VERA). Aplicación de realidad aumentada. European Journal of Investigation in Health, Education and Psychology, 1 (2), 71-84.

Portalès, C. (2008). Entornos multimedia de realidad aumentada en el campo del arte (Tesis doctoral). Facultad de bellas artes de la Universidad Politécnica de Valencia. Recuperado de http://riunet.upv.es/handle/10251/ 3402

Prendes, C. (2014). Propuesta de innovación educativa en un IES basada en una in- 
vestigación exploratoria sobre Realidad Aumentada (Trabajo fin de máster). Recuperado de http://hdl.handle.net/10201/39546

Redondo, E., Fonseca, D., Sánchez, A. \& Navarro, I. (2014). Mobile learning en el ámbito de la arquitectura y la edificación. Análisis de casos de estudio. Revista de Universidad y Sociedad del Conocimiento (RUSC), 11, (1), 152-174. doi:http://doi.dx.org/10.7238/ rusc.v11i1.1844

Reinoso, R. (2012). Posibilidades de la realidad aumentada en educación. En J. Hernández, M. Pennesi, D. Sobrino \& A. Vázquez (Coords). Tendencias emergentes en educación con TIC. (pp.357-400). Barcelona: Editorial espiral.

Reinoso, R. (2013). Módulo 1: Introducción a la realidad aumentada. [Presentación slideshare].Escuela virtual de verano 2013 espiral (eve13). Recuperado de http:// www.slideshare.net/tecnotic

Rice, R. (2009). Augmented vision and the decade of ubiquity. Recuperado de http:// curiousraven.com/future-vision/2009/3/20/ augmented-vision-and-the-decade-ofubiquity.html

Roussou, M. (2004). Learning by Doing and Learning Through Play: An Exploration of Interactivity in Virtual Environments for Children.Computers in Entertainment (CIE) - Theoretical and Practical Computer Applications in Entertainment, 2 (1),1-23. doi: 10.1145/973801.973818

Sánchez, A. (2011). Realidad Aumentada. Una experiencia real. [Archivo de vídeo].I congreso virtual sobre educación y TIC 2011 «La escuela del futuro». Vídeo-presentación. Recuperado de http:// www.youtube.com/watch?v=XojvqauJyNg\& feature=youtu.be
Selltiz, C., Wrightsman, L.S., Cook, S.W. \& Balch, G.I. (1980). Métodos de investigación en las relaciones sociales. Madrid: Rialp S.A.

Silva, J.M. (2013).La realidad aumentada, dentro y fuera del aula. Revista Aula de Secundaria. Didáctica. Tutoría. Gestión. Orientación, (4)19-23.

Vian, R. (2011) Realidad aumentada. Fundamentos y aplicaciones (Tesina). Universidad Politécnica de Valencia. Escuela politécnica superior de Gandía. Recuperado de http://riunet.upv.es/handle/10251/14095
Fecha de recepción: 15-07-2014

Fecha de evaluación: 01-10-2014

Fecha de aceptación: 02-11-2014 